\title{
Tuberculin skin test for control of bovine tuberculosis: limitation history, current challenges and future opportunities
}

\begin{abstract}
Bovine Tuberculosis represents an important constraint to the development of livestock industry especially in the developing countries. In addition, as a zoonotic disease, bovine tuberculosis can cause severe impact on public health. The tuberculin skin test (TST) has been the most widely used method for detecting tuberculosis and for many decades is considered a significant asset for the control of the disease among both animals and human. Despite the intensive worldwide application of TST for control of tuberculosis, an increase in the dieses incidences especially in developing countries is evident and denoted the limitations of the currently used test as a tool for disease control. A replacement antigen for tuberculin purified protein derivatives (PPD) that improve skin test specificity without affecting its sensitivity has been a long-standing research goal. Description of a new reagent - of either single or multiple specific antigenic protein(s) - to replace PPD remains a challenge. The current review will through the light on the limitations of the currently used TST with special reference to the current challenges and future opportunities for developing next-generation reagents for more reliable skin test for control of bovine tuberculosis.
\end{abstract}

Volume 4 Issue 4 - 2017

\author{
Amr M Mohamed ${ }^{1,2}$ \\ 'Department of Animal Medicine, Assiut University, Egypt \\ ${ }^{2}$ Laboratory Medicine Department, Faculty of Applied Medical \\ Science, Umm Al-Qura University, Saudi Arabia

\begin{abstract}
Correspondence: Amr M Mohamed, Current Address: Laboratory Medicine, Collage of Applied Medical Sciences, Umm Al-Qura University, 21955, Saudi Arabia,Tel 00966598936458, Email amrmohamed2004@yahoo.com
\end{abstract}

Received: February 19, 2017 | Published: March 28, 2017

\section{Introduction}

In spite of the great advancement in the diagnosis, treatment and control programs of tuberculosis during the past decades, bovine tuberculosis still represent an imminent threat to the public and considerable obstacles for development of bovine industry especially in developing countries. ${ }^{1}$ All animal species and humans of all age groups are susceptible to the disease. Mycobacterium bovis, a member of the Mycobacterium tuberculosis complex is the causative agent of the disease in cattle. Although Mycobacterium tuberculosis is the main causative agent of human tuberculosis, M. bovis can also infect human causing clinically undistinguishable disease from that of human origin, which makes it an important zoonotic disease of public health concern. ${ }^{2,3}$

Eradication of this zoonotic disease remains an important strategic goal worldwide. The test and slaughter strategy has been widely used in an attempt to control dissemination of the disease either among cattle or to human being. This strategy is based on the tuberculin skin test (TST) for detection of infected or carrier animals. TST has been in use for decades as an asset in the control of bovine tuberculosis. The test is based on the delayed-type hypersensitivity (DTH) reaction elicited by purified protein derivatives (PPD) of Mycobacterium tuberculosis antigens known as tuberculin. Intradermal injection of PPD results in a DTH response in infected animals that peaks 48-72 $\mathrm{h}$ after injection. ${ }^{4}$ Despite the intensive application of TST for control of tuberculosis, an increase in TB incidences especially in developing countries is evident (WHO, 2012). The alarming evidences that denoted limitations in currently used skin testing procedure as an effective tool for control of bovine tuberculosis were the driving power for the scientific community to start investigating other alternatives that could lead to improved efficacy of adopted control measures. ${ }^{5}$ In fact, one cannot dismiss the importance of the TST as a significant asset for the control of tuberculosis as it is extremely simple to perform and does not require extensive training for its application and subsequent scoring of the result. Nevertheless, better skin-test reagent for diagnosis of tuberculosis is on demand and has been the goal for many investigations during the past decade. The current review will reveal the main disadvantages and limitations of the currently used TST as a tool for bovine tuberculosis detection and control. In addition, the current challenges of developing an alternative skin test reagent will be elucidated with special reference to the future opportunities for a reliable next-generation skin test.

\section{History and Limitations of TST}

The tuberculin skin test (TST) has been a useful diagnostic and epidemiological tool for control of bovine tuberculosis for several decades. ${ }^{4}$ However, suboptimal specificity of TST has frequently been reported in somebovine tuberculosis eradication programs. ${ }^{6}$ This suboptimal specificity could be attributed, in part, to the nature of the poorly characterized antigens of the used tuberculin. These antigens are mycobacterial extracts that contain, the antigens comprises of non indigenous components in addition to species-specific components. As a result, the current test has failed to single out the individuals infected by tuberculous mycobactria from those that are immune to Mycobacterium spp as a consequence of exposure to environmental non-tuberculous mycobacteria. ${ }^{7}$ In other words, the reported false positive cases and cross-reactions that occur due to exposure or infection with other non-tuberculous environmental mycobacteria are generally attributed to the fact that the PPD of tuberculin has many antigens shared by other non-tuberculous Mycobacterium species as Mycobacterium avium, M. interacellularae, M. scrofulaceum, M. paratuberculosis, M. kansasii and M. fortuitum. ${ }^{7,8}$ The test questionable specificity complicates its use as a tool for accurate diagnosis and control of both active and latent diseases. Single intradermal comparative tuberculin test (SICTT) was proposed as an alternative field test to overcome this problem. The SICTT compares skin responses to parallel injections of bovine PPD and avian PPD on the two sides of the animal neck. Animals are considered positive when the responses to bovine PPD is greater than parallel responses to avian PPD Nevertheless, although the results of the SICTT constitute 
a good indication of mycobacterial exposure, even this test does not always discriminate between cattle with tuberculosis and those exposed to nonpathogenic organisms. ${ }^{9}$

In order to enhance the success rate of infection detection, an in vitro assay was developed as an alternative method. The assay is based on the detection and quantification of the released IFN-r cytokine when whole blood is cultured with mycobacterial antigens. ${ }^{10}$ Low-molecular-weight antigens such as ESAT-6 and CFP10 have been identified as having great potential as IFN- $\gamma$-inducing antigens of tuberculous mycobacteria. ${ }^{7,11}$ Although, such in vitro immunogenic assay has been shown as a potential alternative for diagnosis of bovine tuberculosis, the test sensitivity is questionable with variable reports ranged from as low as $55 \%$ in some cases and up to $97 \%$ in other cases. ${ }^{12}$ In addition, the test is far from being a simple field screening assay as it needs special laboratory skills and is more expensive when compared to skin testing.

It was shown that the combined use of the tuberculin test together with the IFN- ${ }^{\gamma}$ assay could improve the early detection of $M$. bovisinfected cattle. ${ }^{13}$ However, disadvantages of the combined assay include the possible influence of prior skin testing on the IFN-r assay results. In addition, time factor plays an important role due to the requirement that the blood samples have to be processed for the assay within few hours of collection, which might not always be feasible when testing large population with large geographic distances separate them from the diagnostic laboratory. ${ }^{5,14}$

Rather than relying on in vitro laboratory techniques, the efforts are directed towards development of better antigens to enhance the current field skin testing method and improve its reliability for the control of bovine tuberculosis. The challenge is to increase the specificity of the test reagent with the preservation of its required sensitivity.

\section{Improvement trials between current challenges and future opportunities}

The importance of PPD skin test as a significant asset for the control of tuberculosis cannot be denied. The test is extremely simple to perform and does not require extensive training for its application and subsequent scoring of the result. ${ }^{5,4}$ However, better skin-test reagents for the diagnosis of tuberculosis are needed. A replacement antigen for tuberculin PPD that improve skin test specificity without affecting its sensitivity has been a long-standing research goal. In fact, description of a new reagent - of either single or multiple specific antigenic protein(s) - to replace PPD remains a challenge. Attempts to identify candidate antigens have led to the purification and characterization of many proteins from $M$. tuberculosis and $M$. bovis. Several suchproteins have been investigated as candidate skin test reagents in guinea pig models of tuberculosis. These included ESAT-6, CFP10, MPT64, MPT59, 38-kDa antigen, KatG,MPT32, MTC28, MPT51, MPT70, 19-kDa antigen, MPT63, and DPPD. ${ }^{15}$ ${ }^{20}$ In vitro evaluation of these proteins has given very promising results. For example recombinant ESAT-6and MPT64 were able to differentiate $M$. tuberculosi-infected guinea pigs from $M$. bovis BCG or $M$. avium-sensitized ones. ${ }^{21}$ In addition, some of these reagents namely MPT64 has been reported under phase III clinical trials to evaluate its potential to replace PPD. ${ }^{22}$

In a recent study, our laboratory fractionated locally produced bovine tuberculin PPD with subsequent evaluation of the obtained fractions both in vitro and in vivo in a trial to characterize tuberculous Mycobacterium-specific proteins. The study identified
5 different previously described proteins (CFP10, ESAT6, MPB64, MPB83 and DPPD) that elicited specific in vitro DTH using both LPA and INF- $\gamma$ production of peripheral blood monocytic cells (PBMC) from guinea pig models sensitized with both tuberculous and non tuberculous Mycobacterium species. ${ }^{23}$ Identified proteins included, ESAT-6 and CFP-10, which had been described as being specific tuberculous mycobacterial proteins typically consisting of about 100 amino acids. They belong to a large family of mycobacterial proteins whose members have been identified as potent T-cell antigens. ${ }^{15,24}$ On the other hand, MPB 64, MPB70 and MPB83 are relatively larger antigens that are more abundantly expressed by $M$. bovis than M. tuberculosis. MPT83 (a homologue in M. tuberculosis) is a cell wall associated lip glycoprotein, which is suggested to play a role in adhesion and dissemination based on sequence analysis. ${ }^{18}$ Although the exact function of MPT83 is still unknown, recent studies revealed strong immunogenic properties of the protein with strong cell mediated as well as humeral responses, which suggest a potential protective role of MPT83. ${ }^{19}$ MPB64, MPB70 and MPB83 are proposed to play a role in promoting the initiation of DTH reaction, which can help in production of a more potent DTH response to other dominant antigens. . $^{18,21,25,26}$

DPPD is a major component of the PPD protein mixture complex. It is a small protein composed of 84 amino that induces strong and specific DTH in $M$. tuberculosis-infected guinea pig models. ${ }^{17,27}$ Additional studies suggested DPPD as a potential alternative for PPD ${ }^{16,28}$ Despite the in vitro promising results of those antigenic proteins as potential candidates for better skin testing, in vivo evaluation of those proteins revealed their failure to stimulate measurable DTH comparable to that of conventional PPD in M.bovissensitized guinea pigs. ${ }^{24}$ The failure of these purified single proteins with in vitro promising results to induce measurable DTH comparable to that of conventional PPD in guinea pigs was an obvious challenge. This might be attributed to the small size of the proposed protein $(\sim 5$ to $\sim 25 \mathrm{kDa}$ ) as compared to tuberculin PPD, which may result in rapid diffusion away from the injection site. In fact, over the past 20 years, a wide range of antigenic proteins have been identified and validated as candidate skin test reagents in guinea pig models of tuberculosis, ${ }^{29,30}$ however, efforts to replicate results obtained in guinea pigs for the diagnosis of natural tuberculosis infection have been disappointing and so far no purified antigens have been successfully tested as skin test reagents in cattle or human. ${ }^{20,31,32}$

Therefore, new strategies need to be employed to discover more sensitive and specific skin test antigens. A single antigen may not effectively replace the PPD, alternatively, a cocktail of several DTH- inducing antigens may be required for the optimal TST..$^{20,33,34}$ Innovating of a new multiple-protein antigenic reagent to replace PPD would greatly enhance detection power of both active and latent tuberculosis and hence benefit worldwide control programs. In this regards, the recent identification of the molecular composition of PPD facilitated the development of a more refined reagent. In addition, proteomic studies identified new dominant proteins in PPD, from which, GroES, GroEL2, and DnaK were described as the three most abundant. ${ }^{35,36}$ Recently, 2 formulations of these dominant proteins with other tuberculous mycobacterium-specific antigens (DnaK/GroEL2/ Rv0685) and (DnaK/GroEL2/Rv0009) were proposed as being capable of inducing DTH responses equivalent to PPD in tuberculosis guinea pig model [30]. Understanding of the DTH response driven by these defined proteins as compared to that of the more complex tuberculin PPD can contribute to the innovation of a sensitive and more specific reagent. 
The obvious difference in physical composition of tuberculin PPD as compared to proposed recombinant proteins is another important factor that needs to be considered while looking for alternative reagents. PPD is a complex of protein and non-protein components, while the proposed antigenic proteins are single proteins in a more purified state. In addition, the crude nature of tuberculin PPD and the presence of pro-inflammatory factors, which are missing from the purified recombinant proteins, is proposed to play a possible stimulatory influence of cytokine and chemokine networks that promote the DTH reaction elicited in response to main antigenic proteins. ${ }^{37}$ It has long been recognized that poor humoral and cellmediated immune responses usually occur when antigenic proteins injected without suitable adjuvant. ${ }^{38}$ Adjuvants stimulate the release of the pro-inflammatory cytokines (TNF- $\alpha$ and IL-1), which in turn stimulate the migration of dendritic cells (DC) to the antigen injection site. Subsequently, the presentation and recognition of the antigen by memory $\mathrm{T}$ cells stimulate an influx of monocytes, macrophages, and $\mathrm{T}$ cells to the antigen injection site. ${ }^{38}$ Absence of adjuvant with the subsequent lack of pro-inflammatory conditions with the low level of DC migration that occurs under non-inflammatory conditions usually result in insufficient presentation of the antigen. ${ }^{38}$ Therefore, the poor performance of selected proteins as a skin test reagent in cattle could be attributed in part to the absence of a suitable proinflammatory stimulus. Bacterial lipoproteins and lipopeptides from a diverse range of bacteria, including Escherichia coli, Borrelia burgdorferi, Treponema pallidum, Mycoplasma fermentans, and $M$. tuberculosis, have been shown to induce pro-inflammatory cytokine production from monocytes and macrophages through triggering of the innate pattern recognition signal Toll-like receptor $2 .{ }^{39}$ Inclusion of these lipoproteins in any proposed mixture of antigenic proteins could enhance their DTH reactions and produce better next-generation skin test reagents that can provide simple, rapid and accurate detection of both active and latent TB infection.

\section{Conclusion}

In conclusion, the importance of PPD skin test as a significant asset for the control of bovine tuberculosis cannot be denied. Therefore, current improvement efforts are directed towards keeping the simplicity of this field test while enhancing its specificity via looking for better antigen(s) to serve as a reliable candidate reagent for the test. Although research efforts over the past decade succeeded in isolation and production of several promising proteins that elicited specific antigenicity and produced interesting and encouraging in vitro results, no single reagent yet has provided reliable diagnostic results either in vivo or under real field conditions. Observing the different nature of DTH reaction in response to the proposed recombinant purified protein(s) in one hand, and to the more complex tuberculin PPD on the other hand, taking in consideration the obvious difference in PPD physical composition as compared to recombinant proteins would suggest the need for different strategy. The new strategy should be directed towards using antigenic protein cocktails rather than single proteins with inclusion of pro-inflammatory reagents to trigger the DTH reaction and make it more visible and detectable.

\section{Conflicts of Interest}

The authors declare no conflict of interest.

\section{Acknowledgments}

None.

\section{Funding}

None.

\section{References}

1. World Health Organization. Neglected zoonotic diseases. World Health Organization Report. 2013.

2. Etchechoury I, Valencia GE, Morcillo N, et al. Molecular Typing of Mycobacterium bovis Isolates in Argentina: First Description of a Person-to-Person Transmission Case. Zoonoses Pub Hlth. 2010;57(6):375-381.

3. O Reilly LM, Daborn CJ. The epidemiology of Mycobacterium bovis infections in animals and man: a review. Tuber Lung Dis. 1995; 1(Suppl 76):1-46.

4. Monaghan ML, Doherty ML, Collins JD, et al. The tuberculin test. Vet Microbiol. 1994;40(1-2):111-124.

5. Gormley E, Doyle MB, McGill K, et al. The effect of the tuberculin test and the consequences of a delay in blood culture on the sensitivity of a gamma-interferon assay for the detection of Mycobacterium bovis infection in cattle. Vet Immunol Immunopathol. 2004;102(4):413420

6. Schiller I, Vordermeier HM, Waters WR, et al. Comparison of tuberculin activity using the interferon-gamma assay for the diagnosis of bovine tuberculosis. Vet Rec. 2010;167(9):322-326.

7. Pollock JM, Andersen P. The potential of the ESAT-6 antigen secreted by virulent mycobacteria for specific diagnosis of tuberculosis. $J$ Infect Dis. 1997;175(5):1251-1254.

8. Huebner RE, Schein MF, Bass JB, et al, The tuberculin skin test. Clin Infect Dis. 1993;17(6):968-975.

9. Whelan AO, Hope JC, Howard CJ, et al. Modulation of the bovine delayed-type hypersensitivity responses to defined mycobacterial antigens by a synthetic bacterial lipopeptide. Infect Immun. 2003;71(11):6420-6425.

10. Wood PR, Jones SL. BOVIGAM: an in vitro cellular diagnostic test for bovine tuberculosis. Tuberculosis (Edinb). 2001;81(1-2):147-155.

11. Van Pinxteren LA, Ravn P, Agger EM, et al. Diagnosis of tuberculosis based on the two specific antigens ESAT-6 and CFP10. Clin Diagn Lab Immunol. 2000;7(2):155-160.

12. Monaghan MQ, Quinn PJ, Kelly AP, Mcgill K, et al. A pilot trial to evaluate the G-interferon assay for the detection of Mycobacterium bovis infected cattle under Irish conditions. Ir Vet J. 1997;50(4):229322 .

13. Neill SD, Cassidy J, Hanna J, et al. Detection of Mycobacterium bovis infection in skin test-negative cattle with an assay for bovine interferon-gamma. Vet Rec. 1994;135(6):134-135.

14. Ryan TJ, Buddle BM, De Lisle GW. An evaluation of the gamma interferon test for detecting bovine tuberculosis in cattle 8 to 28 days after tuberculin skin testing. Res Vet Sci. 2000;69(1):57-61.

15. Aagaard C, Govaerts M, Meikle V, et al. Optimizing antigen cocktails for detection of Mycobacterium bovis in herds with different prevalences of bovine tuberculosis: ESAT6-CFP10 mixture shows optimal sensitivity and specificity. J Clin Microbiol. 2006;44(12):4326-4335.

16. Campos Neto A, Rodrigues Junior V, Pedral-Sampaio DB, et al. Evaluation of DPPD, a single recombinant Mycobacterium tuberculosis protein as an alternative antigen for the Mantoux test. Tuberculosis (Edinb). 2001;81(5-6):353-358.

17. Coler RN, Skeiky YA, Ovendale PJ, et al. Cloning of a Mycobacterium tuberculosis gene encoding a purifed protein derivative protein that elicits strong tuberculosis-specific delayed-type hypersensitivity. $J$ Infect Dis. 2000;182(1):224-233. 
18. Hewinson RG, Michell SL, Russell WP, et al. Molecular characterization of MPT83: a seroreactive antigen of Mycobacterium tuberculosis with homology to MPT70. Scand J Immunol. 1996;43(5): 490-499.

19. Kao FF, Mahmuda S, Pinto R, et al. The Secreted Lipoprotein, MPT83, of Mycobacterium tuberculosis Is Recognized during Human Tuberculosis and Stimulates Protective Immunity in Mice. PLOS ONE. 2012;7(5): e34991.

20. Lyashchenko K, Manca C, Colangeli R, et al. Use of Mycobacterium tuberculosis complex-specific antigen cocktails for a skin test specific for tuberculosis. Infect Immun. 1998;66(8):3606-3610.

21. Wiker HG, Lyashchenko KP, Aksoy AM, et al. Immunochemical characterization of the MPB70/80 and MPB83 proteins of Mycobacterium bovis. Infect Immun. 1998;66(4):1445-1452

22. Elhay MJ, Oettinger T, Andersen P. Delayed-type hypersensitivity responses to ESAT-6 and MPT64 from Mycobacterium tuberculosis in the guinea pig. Infect Immun. 1998;66(7):3454-3456.

23. Wang Z, Potter BM, Gray AM, et al. The solution structure of antigen MPT64 from Mycobacterium tuberculosis defines a new family of betagrasp proteins. J Mol Biol. 2007;366(2):375-381.

24. Mohamed AM, Ashshi AM, Refaat BA, et al. Towards Improvement of Tuberculin Skin Test: five Potential Antigenic Proteins Elicited in vitro Specific Immune Reaction Against Tuberculous Mycobacterium Spicies Using Sensitized Guinea Pig Models. J Anim Vet Advac1. 2015;4(10):281-292.

25. Lightbody KL, Ilghari D, Waters LC, et al. Molecular features governing the stability and specificity of functional complex formation by Mycobacterium tuberculosis CFP-10/ESAT-6 family proteins. J Biol Chem. 2008;283(25):17681-17690.

26. Whelan AO, Clifford D, Upadhyay B, et al. Development of a skin test for bovine tuberculosis for differentiating infected from vaccinated animals. J Clin Microbiol. 2010;48(9):3176-3181.

27. Whipple DL, Palmer MV, Slaughter RE, et al. Comparison of purified protein derivatives and effect of skin testing on results of a commercial gamma interferon assay for diagnosis of tuberculosis in cattle. $J$ Vet Diagn Invest. 2001;13(2):117-122.

28. Liu C, Flamoe E, Chen HJ, et al. Expression and purification of immunologically reactive DPPD, a recombinant Mycobacterium tuberculosis skin test antigen, using Mycobacterium smegmatis and Escherichia coli host cells. Can J Microbiol. 2004;50(2):97-105.
29. Reed SG, Campos-Neto A. Vaccines for parasitic and bacterial diseases. Curr Opin Immunol. 2003;15(4):456-460.

30. Yang H, Troudt J, Grover A, et al. Three protein cocktails mediate delayedtype hypersensitivity responses indistinguishable from that elicited by purified protein derivative in the guinea pig model of Mycobacterium tuberculosis infection. Infect Immun. 2011;79(2):716-723.

31. Jones GJ, Khatri BL, Garcia Pelayo MC, et al Development of an unbiased antigen-mining approach to identify novel vaccine antigens and diagnostic reagents for bovine tuberculosis. Clin Vaccine Immunol. 2013;20(11):1675-1682.

32. Yang H, Kruh Garcia NA, et al. Purified protein derivatives of tuberculin - past, present, and future. FEMS Immunol Med Microbiol. 2012;66(3):273-280.

33. Oettinger T, Holm A, Mtoni IM, et al. Mapping of the delayedtype hypersensitivity-inducing epitope of secreted protein MPT64 from Mycobacterium tuberculosis. Infect Immun. 1995;63(12):46134618.

34. Rhodes SG, Gavier Widen D, Buddle BM, et al. Antigen specificity in experimental bovine tuberculosis. Infect Immun. 2000;68(5):2573-2578.

35. Borsuk S, Newcombe J, Mendum TA, et al. Identification of proteins from tuberculin purified protein derivative (PPD) by LC-MS/MS Tuberculosis (Edinb). 2009;89(6):423-430

36. Cho YS, Dobos KM, Prenni J, et al. Deciphering the proteome of the in vivo diagnostic reagent "purified protein derivative" from Mycobacterium tuberculosis. Proteomics. 2012;12(7):979-991.

37. Pollock JM, McNair J, Bassett H, et al. (2003) Specific delayed-type hypersensitivity responses to ESAT-6 identify tuberculosis-infected cattle. J Clin Microbiol. 2003;41(5):1856-1860.

38. Jenkins MK, Khoruts A, Ingulli E, et al. In vivo activation of antigenspecific CD4 T cells. Annu Rev Immunol. 2001;19: 23-45.

39. Brightbill HD, Libraty DH, Krutzik SR, et al. Host defense mechanisms triggered by microbial lipoproteins through toll-like receptors. Science. 1999;285(5428):732-736. 